\begin{tabular}{|c|c|}
\hline Title & $\begin{array}{l}\text { Development of Small Dielectric Lens for Slot A ntenna U sing Topology Optimization with Normal ized Gaussian } \\
\text { Network }\end{array}$ \\
\hline Author(s) & Itoh, Keiichi; Nakaj ima, Haruka; Matsuda, Hideaki; T anaka, Masaki; Igarashi, Hajime \\
\hline Citation & $\begin{array}{l}\text { IEICE transactions on electronics, E101.C(10), } 784.790 \\
\text { https://doi.org/10.1587 transele.E101.C.784 }\end{array}$ \\
\hline Issue Date & $2018-10$ \\
\hline Doc URL & http:/hdl.handle.net/2115/72063 \\
\hline Rights & copyright@2018 IEICE \\
\hline Type & article \\
\hline File Information & e101-c_10_784.pdf \\
\hline
\end{tabular}

Instructions for use 


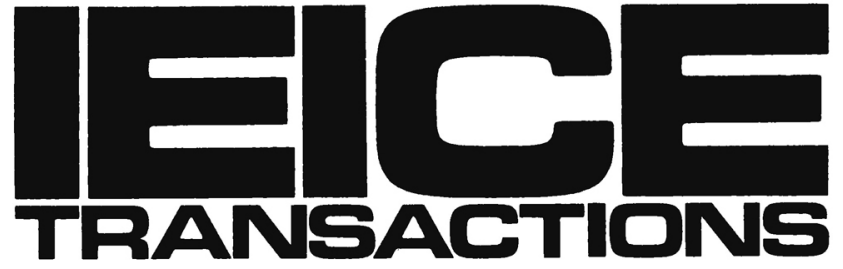

\section{on Electronics}

VOL. E101-C NO. 10

OCTOBER 2018

The usage of this PDF file must comply with the IEICE Provisions on Copyright.

The author(s) can distribute this PDF file for research and educational (nonprofit) purposes only.

Distribution by anyone other than the author(s) is prohibited. 


\title{
Development of Small Dielectric Lens for Slot Antenna Using Topology Optimization with Normalized Gaussian Network
}

\author{
Keiichi ITOH $^{\dagger \text { a) }}$, Member, Haruka NAKAJIMA ${ }^{\dagger}$, Hideaki MATSUDA ${ }^{\dagger}$, Nonmembers, Masaki TANAKA ${ }^{\dagger}$, \\ and Hajime IGARASHI ${ }^{\dagger \dagger}$, Members
}

\begin{abstract}
SUMMARY This paper reports a novel 3D topology optimization method based on the finite difference time domain (FDTD) method for a dielectric lens antenna. To obtain an optimal lens with smooth boundary, we apply normalized Gaussian networks (NGnet) to 3D topology optimization. Using the proposed method, the dielectric lens with desired radiation characteristics can be designed. As an example of the optimization using the proposed method, the width of the main beam is minimized assuming spatial symmetry. In the optimization, the lens is assumed to be loaded on the aperture of a waveguide slot antenna and is smaller compared with the wavelength. It is shown that the optimized lens has narrower beamwidth of the main beam than that of the conventional lens.

key words: topology optimization, normalized Gaussian networks, micro genetic algorithm, FDTD method
\end{abstract}

\section{Introduction}

Because dielectric lens antennas realize high aperture efficiency, they are often used as highly efficient directional antennas [1]-[4]. As a lens for the lens antenna which converges the main beam, the extended hemispherical lens [1], [3] and spherical lens [2], [4] have been proposed. It is expected that not only a directional antenna with a narrow beamwidth but also a fan beam antenna with a wide angle can be realized if it is possible to realize desired radiation patterns merely by loading the dielectric lens. However, there has been no systematic design method to realize the lens antenna which has the desired beam pattern.

The topology optimization is promising method as a dielectric shape design method required for designing the lens antenna mentioned above. There are some prior studies on the topology optimization in the field of antenna and propagation. For example, the topology optimization based on the adjoint variable method (AVM) and density method has been shown effective for design of dielectric resonator antennas (DRA) [5]. This optimization method works fast because it is based on the deterministic method which needs relatively small function calls for field computations. However, the deterministic search falls into local minima depending on the initial guess if the landscape is

\footnotetext{
Manuscript received February 15, 2018.

Manuscript revised May 31, 2018.

$\dagger$ The authors are with National Institute of Technology, Akita College, Akita-shi, 011-8511 Japan.

${ }^{\dagger}$ The author is with Graduate School of Information Science and Technology, Hokkaido University, Sapporo-shi, 060-0808 Japan.

a)E-mail: itok@akita-nct.ac.jp

DOI: 10.1587/transele.E101.C.784
}

multimodal. In others, the topology optimization of the optical waveguide devices has been reported [6]. In [6], the topology optimization of the refractive index distribution by the density method is performed, and the function expansion method using Fourier series is introduced to eliminate the gray area. While the desired property can be obtained with this method, the sensitivity analysis is required to optimize the parameters of the basis function.

In this study, due to design a 3D dielectric lens for antenna, the topology optimization based on the On/Off method is adopted in conjunction with the finite difference time domain (FDTD) method. In this method, the FDTD cells in the design region has one of the two states: the dielectric and air. For this reason, the gray level is not appeared. Moreover, we determine the cell state using the genetical algorithm (GA). Thus, the proposed method does not only require the sensitivity analysis, but also enjoys high search ability being independent from the initial guess. It is known that the On/Off method tends to result in complicated resultant shapes sometimes including checker board patterns, for which we have difficulties in manufacturing actually [7]. This problem comes from the fact that the cell states are independently determined. It has been shown that this problem can be relaxed by using the normalized Gaussian network (NGnet) [8] in the optimization of electric motors. In this method, the cell states are no longer independent but are determined from the value of the shape function represented by the NGnet. We adopt here NGnet for the 3D optimization of lens antenna. The topology optimization based on the NGnet and stochastic algorithm has never been applied to three-dimensional problems.

In this paper, we show that the present method is effective for three-dimensional design problems, especially design of three-dimensional dielectric antennas. The present method has advantages over the conventional method: it employs GA which scarcely depends on the initial guess and also it easily finds the distribution with holes. As a design example, the shape of the small dielectric lens loaded on a waveguide slot antenna is optimized to narrow the beamwidth of the main beam. The FDTD cell states, \{dielectric, air\}, in the design region are determined by GA so that the beamwidth becomes minimum. Then a dielectric lens is manufactured on the basis of the optimized result and its property is compared with the computed results. 


\section{Topology Optimization Method}

\subsection{On/Off Method Based on NGnet}

In the general topology method, as shown in Fig. 1, the elements are usually set individually. In contrast, the On/Off setting method using a Gaussian function with suitable variance is expected to ease the grouping of several elements. The outline and the flow of the On/Off method based on the NGnet are presented in Fig. 2 [9]. As an example, the case in which three Gaussian functions $G(x)$ are arranged linearly on the $x$ axis, as shown in Fig. 2 (a), is described. First, as shown in Fig. 2 (b), the normalized Gaussian function $b(x)$ is calculated for input $x$. The range of $b(x)$ becomes $[0,1]$ because it is normalized by the sum of the Gaussian functions for each input $x$. Next, each $b(x)$ is multiplied by the weighting coefficient $w$. The sum of product $w \times b(x)$ is calculated for each input $x$. The range of $w$ is set as $[-1,1]$. Finally, if the variance is chosen appropriately, then output $y(x)$ is presumed to change smoothly with respect to input $x$, as shown in Fig. 2 (c).

Using the obtained output $y(x), \mathrm{On} / \mathrm{Off}$ states are set as follows: $x$ is "On" when $y(x) \geq 0 ; x$ is "Off" when $y(x)<$ 0 . The Gaussian function $G_{k}(x)$, the normalized Gaussian function $b_{i}(x)$, and the output $y(x)$ are defined as follows.

$$
\begin{aligned}
& G_{k}(\boldsymbol{x})=\frac{1}{(2 \pi)^{D / 2}|\Sigma|^{1 / 2}} \times \exp \left[-\frac{1}{2}\left(\boldsymbol{x}-\boldsymbol{\mu}_{k}\right)^{T} \Sigma_{k}^{-1}\left(\boldsymbol{x}-\boldsymbol{\mu}_{k}\right)\right] \\
& b_{i}(\boldsymbol{x})=\frac{G_{i}(\boldsymbol{x})}{\sum_{k=1}^{N} G_{k}(\boldsymbol{x})}
\end{aligned}
$$

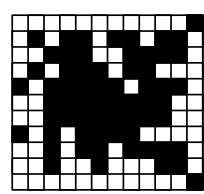

(a) Conventional

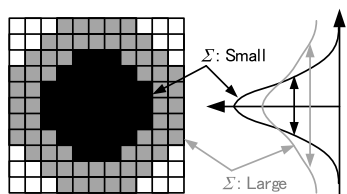

(b) Gaussian function
Fig. 1 Image of On/Off setting using Gaussian function.

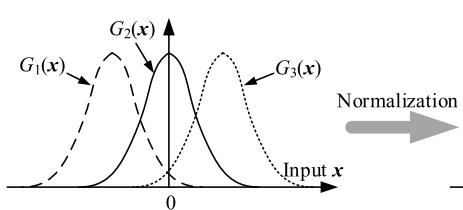

(a) Gaussian output $G(\boldsymbol{x})$

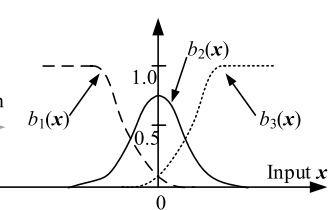

(b) Basis output $b(\boldsymbol{x})$

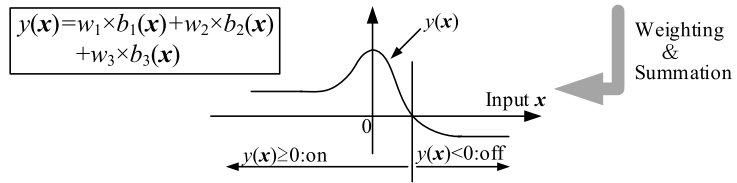

(c) NGnet output $y(x)$

Fig. 2 Outline of On/Off setting method using NGnet.

$$
y(\boldsymbol{x})=\sum_{k=1}^{N} w_{i} b_{i}(\boldsymbol{x})
$$

Therein, $\boldsymbol{x}$ is position vector, $w_{i}$ is the weighting coefficient, $N$ stands for the number of the Gaussian functions, $D$ signifies the dimension of input $x$, and $\boldsymbol{\mu}_{k}$ and $\Sigma_{k}$ respectively denote the center vector and the covariance matrix of the Gaussian function $k$. Three of $w_{i}, \boldsymbol{\mu}_{k}$, and $\Sigma_{k}$ are the parameters which should be optimized.

\subsection{Topology Optimization Using NGnet}

In this study, we choose to optimize only the weighting coefficient among three parameters, expecting the solution to converge easily. To optimize the weighting coefficient, an evolutional calculation method is adopted: the micro genetic algorithm $(\mu \mathrm{GA})$ [4], [10]. In this optimization, the weighting coefficient is treated as the gene in the $\mu \mathrm{GA}$. To obtain a smoother lens shape, the gene is given not as the bit-coded type but as the real-coded type.

The objective function $\mathrm{OF}$ of the $\mu \mathrm{GA}$ is evaluated using FDTD calculations. The number of individuals is set to 5. As the generation progressed, it is presumed that the absolute value of the weighting coefficient exceeds 1 by crossover. Therefore, the weighting coefficient in each generation is normalized so that its range is always modified to $[-1,1]$.

\section{3D Topology Optimization of Dielectric Lens}

\subsection{Analysis Model}

As shown in Fig. 3, the lens design region is placed on the aperture of the 1-slot type waveguide slot antenna. The relative permittivity in the design region is 2.2 in the case of the dielectric and 1.0 in the case of air. The Gaussian basis, which is $3 \times 3 \times 3$ case, is arranged in $3 \mathrm{D}$ as shown in Fig. 3 (b). The analysis conditions are presented in Table 1.

To improve the directivity by the dielectric lens, the dielectric lens shape is optimized to minimize the main beam beamwidth. Although to evaluate the beamwidth requires the far field calculation [11], the computational load becomes high. In addition, the $\mu \mathrm{GA}$ is known to require a long calculation time. To resolve this calculation cost problem, the proposed optimization is calculated using a super-

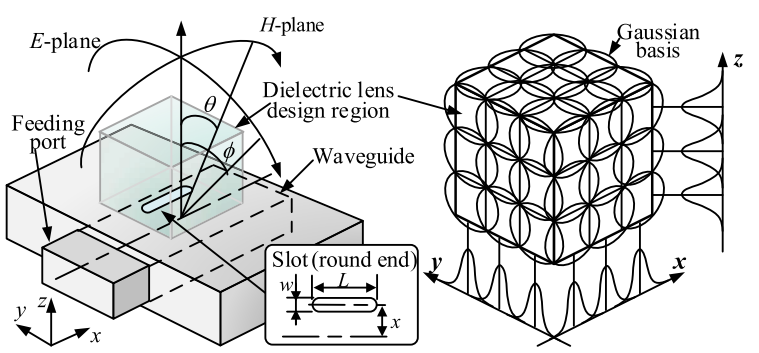

Fig. 3 Outline of analysis model and placement of Gaussian basis in the dielectric lens design region. 
Table 1 Analysis conditions of lens and antenna.

\begin{tabular}{ll}
\hline Parameters & Conditions \\
\hline Cell size & $0.5 \mathrm{~mm}$ \\
FDTD analysis region & $94 \times 130 \times 90$ cells \\
Absorbing boundary condition & PML $(8$ layers $)$ \\
Lens design region & $40 \times 40 \times 40$ cells $(20 \times 20 \times 20 \mathrm{~mm})$ \\
Relative permittivity & $2.2($ dielectric $) / 1.0($ air $)$ \\
Number of Gaussian basis & $2 \times 2 \times 2,3 \times 3 \times 3,4 \times 4 \times 4,7 \times 7 \times 7$ \\
Slot shape & Round ends \\
Slot length $L$ & 25 cells $(12.5 \mathrm{~mm})$ \\
Slot width $w$ & 4 cells $(2.0 \mathrm{~mm})$ \\
Slot offset $x$ & 15 cells $(7.5 \mathrm{~mm})$ \\
Waveguide inner size & $23.0 \times 10.0 \mathrm{~mm}($ WRJ-10 Standard $)$ \\
Waveguide excitation mode & TE 10 mode \\
Waveguide termination & Reflectless \\
Incident source & Continuous wave, $12 \mathrm{GHz}$ \\
Wavelength & 25.0 mm \\
\hline
\end{tabular}

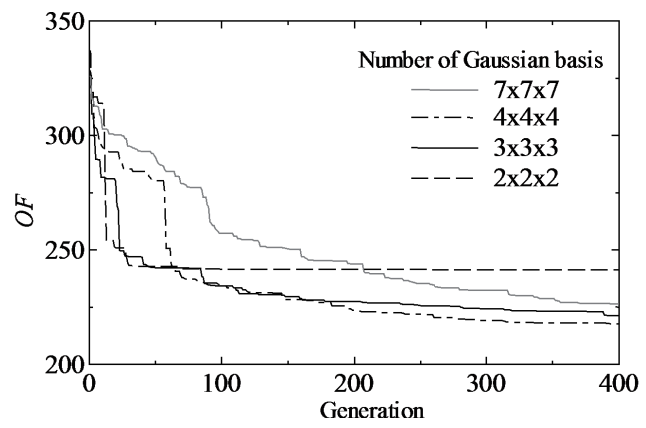

Fig. 4 Comparison of changes in $O F$.

computer system (SR16000/M1; Hitachi Ltd.) at Hokkaido University.

\subsection{Optimization Results}

In this section, we examine a suitable number of Gaussian bases. To reduce the calculation time, the far field radiation patterns is calculated from calculation results of the near field. By calculating $-20 \mathrm{~dB}$ beamwidth $B W_{H}$ and $B W_{E}$ from the $H$-plane and $E$-plane far field radiation patterns, the sum of both beamwidths is set as the objective function $O F$.

The relation between the number of the Gaussian basis and the convergence speed of the $O F$ is examined by changing the number of bases. The variance value $\Sigma$ is also appropriately changed according to the number of bases. As shown in Fig. 4, the changes in the $O F$ show that, when the number of bases is $3 \times 3 \times 3$ or more, the solution converges sufficiently. In addition, as the number of bases increases, the lens shape can be expected to become more complicated, but the convergence speed becomes slower.

\subsection{Design of Narrow Angle Lens}

Based on the discussion presented above, the design of the narrow angle lens is performed. The topology optimization in case of $4 \times 4 \times 4$ bases is calculated up to 1000 generations. The far field radiation patterns are calculated according to

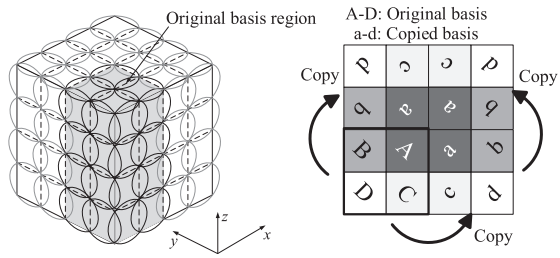

$\begin{array}{ll}\text { (a) Design region } & \text { (b) Copy image (top view) }\end{array}$

Fig. 5 Placement of Gaussian basis in the dielectric lens design region.

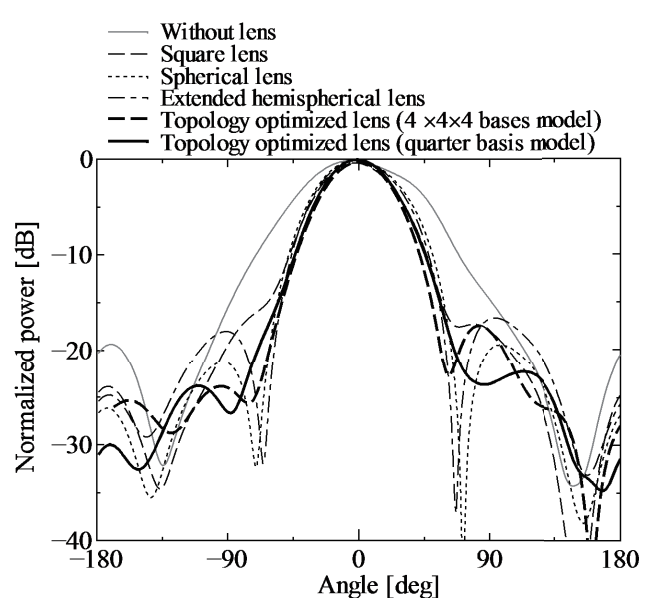

Fig. $6 \quad H$-plane far field radiation patterns.

[11]. The objective function $O F$ is set to the sum of the $-10 \mathrm{~dB}$ beamwidth $B W_{H}$ and $B W_{E}$. The narrow angle lens design is realized to minimize $O F$. The calculation time took about 17 hours when using the supercomputer system.

Generally, it is considered that the symmetric lens shape is easy to manufacture in comparison with the asymmetric lens shape. Therefore, On/Off state setting using the NGnet is also performed only for a quarter region, as shown in Fig. 5. On/Off states in other regions are set by copying of the original region. In both topology optimizations of the full $4 \times 4 \times 4$ bases model and a quarter basis model, the variance value is set to $\Sigma=0.0022$.

Figures 6 and 7 show both far field radiation patterns with the optimized lenses. For comparison, the radiation patterns with the conventional lens are also shown. The beamwidth with both topology optimized lenses are greatly improved in the $E$ - plane. The beamwidth of each lens is presented in Table 2. These results show that the beamwidth with the topology optimized lenses is reduced to about half of that without a lens, and to about $15 \%$ of that with conventional lenses. It is observed that the far-field radiation patterns with the topology optimized lens have little chenges in the frequency range from 11.8 to $12.2 \mathrm{GHz}$.

As shown in Fig. 8 (a), the lens shape for the case of $4 \times 4 \times 4$ bases model becomes asymmetrical. In contrast, as shown in Fig. 8(b), the symmetrical shape and the narrow beamwidth are obtained for the case of a quarter basis model, which shows that the proposed method has good design ability. In addition, the reflection coefficient $S_{11}$ and 
Table 2 Optimization results.

\begin{tabular}{|c|c|c|c|c|c|}
\hline Lens shape & $\begin{array}{c}B W_{H}+B W_{E} \\
(O F)[\mathrm{deg}]\end{array}$ & $\begin{array}{l}B W_{H} \\
{[\mathbf{d e g}]}\end{array}$ & $\begin{array}{c}B W_{E} \\
{[\mathbf{d e g}]}\end{array}$ & $\begin{array}{c}S_{11} \\
{[\mathbf{d B}]}\end{array}$ & $\begin{array}{c}S_{21} \\
{[\mathbf{d B}]}\end{array}$ \\
\hline Without lens & 370.42 & 140.47 & 229.94 & -20.47 & -1.848 \\
\hline Square lens & 219.52 & 95.94 & 123.58 & -31.16 & -0.445 \\
\hline Sphere lens & 214.33 & 99.35 & 114.98 & -27.43 & -0.741 \\
\hline Extended hemisphere lens & 222.71 & 94.64 & 128.07 & -29.26 & -0.576 \\
\hline $\begin{array}{l}\text { Topology optimized lens } \\
(4 \times 4 \times 4 \text { bases model) }\end{array}$ & 184.32 & 86.90 & 97.42 & -29.23 & -0.553 \\
\hline $\begin{array}{l}\text { Topology optimized lens } \\
\text { (quarter basis model) }\end{array}$ & 185.02 & 89.77 & 95.25 & -24.49 & -1.058 \\
\hline
\end{tabular}

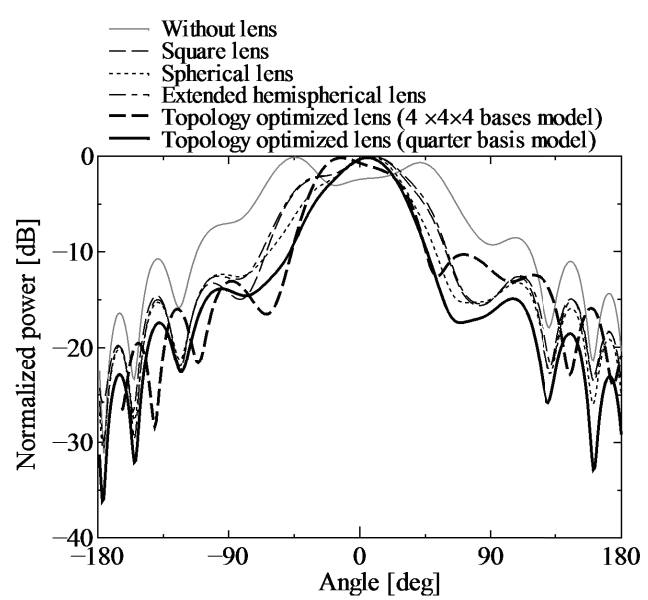

Fig. $7 \quad E$-plane far field radiation patterns.

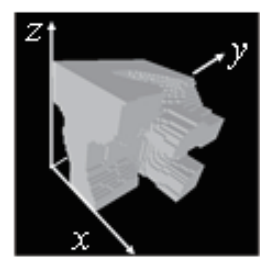

(top side view)

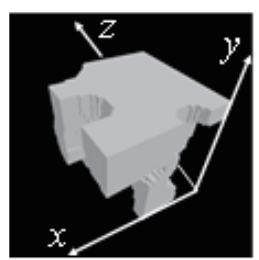

(Bottom side view)

(a) $4 \times 4 \times 4$ bases model

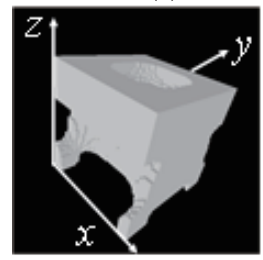

(top side view)

(b) Quarter basis model

Fig. 8 Optimized lens shapes.

the transmission coefficient $S_{21}$ of each conditions are summarized in Table 2. When loading the lens, it is found that $S_{11}$ and $S_{21}$ are changed. Among all lenses, both coefficents in case of a quarter basis model are close to those without the lens as compared with other lens antennas, because this lens is not touch the slot, as shown in Fig. 8 (b).

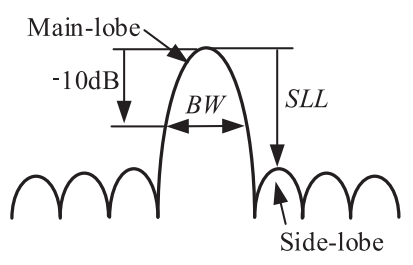

Fig.9 Definitions of $B W$ and $S L L$.

\section{Beam-Forming Using Multi Objective Optimization}

\subsection{Optimization Results}

Next, for radar applications, the narrow angle lens design for only the $H$-plane is performed. When only the $H$ plane beamwidth is minimized, the side-lobe level is enhanced. Therefore, the side-lobe suppression scheme is also required. It is necessary to satisfy two objective functions of minimizing $-10 \mathrm{~dB}$ beamwidth $B W$ and maximizing the side-lobe level ratio $S L L$, which represents the absolute value of the difference between the maximum value of the main-lobe and the maximum value of the side-lobe, as shown in Fig. 9. the multi-objective optimization using a quarter basis model is conducted according to the following objective function.

$$
O F=\frac{B W}{B W_{0}}+w \times \frac{S L L_{0}}{S L L}
$$

In that equation, $w$ is a weighting coefficient; $B W_{0}$ and $S L L_{0}$ are reference levels: $B W_{0}$ is set to the beamwidth without lens, and $S L L_{0}$ is set to 1.0 .

In Fig. 10, $O F_{1}$ and $O F_{2}$ respectively denote optimization results with $w=0.0$ and $w=0.1$. Optimization of the side-lobe level is not considered in $O F_{1}$. The $E$-plane far field radiation patterns with both optimized lenses are almost identical. In contrast, the side-lobe level in the $H$ plane far field radiation patterns is drastically different. The $S L L$ of $O F_{2}$ is improved from $8.34 \mathrm{~dB}$ to $20.80 \mathrm{~dB}$ as compared with $S L L$ of $O F_{1}$. The relation between $B W$ and $S L L$ for each weighting coefficient $w$ is shown in Table 3, which shows that a tradeoff relation between $B W$ and $S L L$.

Optimization results reveal that the proposed method can realize beam-forming of the antenna. In addition, the symmetrical shapes are obtained, as shown in Fig. 11. The topology optimized lens in Fig. 11 (a) is not observed the 


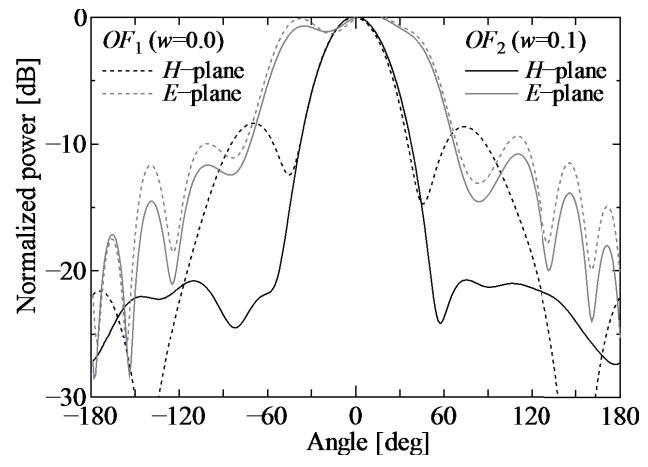

Fig. $10 \quad H$-plane far field radiation patterns.

Table 3 Optimization results.

\begin{tabular}{cccc}
\hline Weighting coefficient & $B W[\mathbf{d e g}]$ & $S L L[\mathbf{d B}]$ & Remarks \\
\hline$w=0.0$ & 73.34 & 8.34 & $O F_{1}$ \\
$w=0.1$ & 75.27 & 20.80 & $O F_{2}$ \\
$w=0.5$ & 78.40 & 25.93 & \\
$w=1.0$ & 82.94 & 29.50 & \\
& & &
\end{tabular}

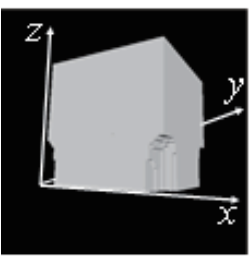

(side view)

(a) $O F$

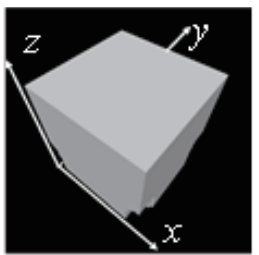

(top side view)

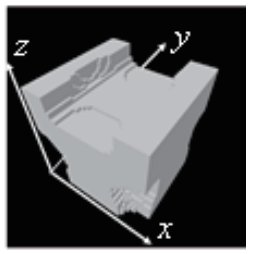

(top side view) (b) $\mathrm{OF}_{2}$

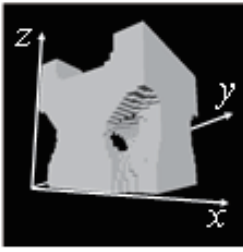

(side view)
Fig. 11 Optimized lens shapes.

hole inside the lens. Figure 11 (b), on the contrary, shows that the topology optimized lens has a hollow structure in the center and a concave structure on the top. In the next section, we consider the relationship between the shape of the topology optimized lens and the radiation properties.

\subsection{Consideration}

To discuss the shape of the topology optimized lens, we analyze the phase delay in the dielectric region. Figure 12 shows the cross section of each lenses and analysis planes \#1 to \#4 in which the phase delay is calculated. By calculating the phase delay from the reference in the dielectric region, the wavefront in each analysis planes can be presumed. In addition, by comparing with the phase delay in the extended hemispherical lens, which is the conventional lens, we clarify the features of the topology optimized lens.

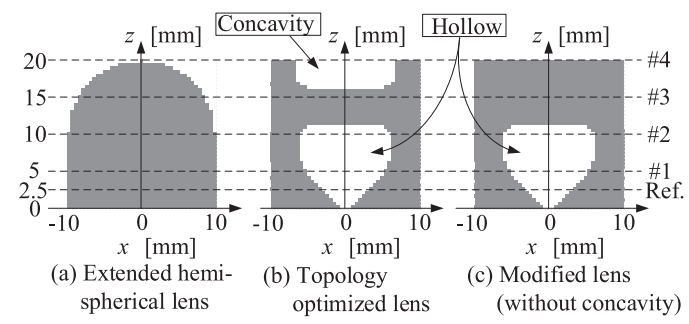

Fig. 12 Cross section of each lens and analysis plane of phase.

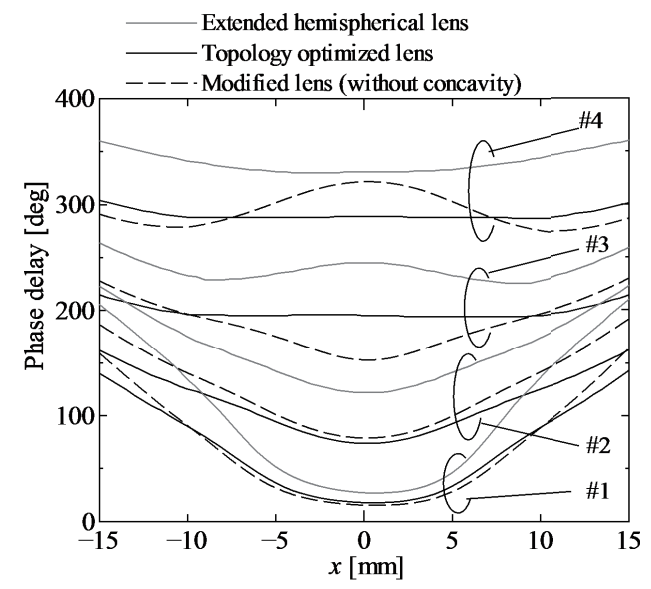

Fig. 13 Phase distribution of each lens in analysis plane.

As shown in the analysis plane \#2 in Fig. 13, wavefronts of both lenses become substantially spherical wave. In the extended hemispherical lens, the phase delay around the lens center in the analysis plane \#3 becomes large. Finally, since the phase delay in the analysis plane \#4 becomes uniform as compared with that in the analysis plane \#2, it is confirmed that the convergence effect is obtained. In contrast, in the topology optimized lens, the equiphase plane is observed over the range of $\pm 10 \mathrm{~mm}$ in both analysis planes \#3 and \#4. As a result, since the parallel wavefront is obtained, it is considered that the high directivity is realized in the topology optimized lens.

Next, the modified lens which is filled with the dielectric in the concave of the topology optimized lens, as shown in Fig. 12 (c), is modeled, and the phase delay is calculated, as shown in Fig. 13. In the analysis plane \#4 without the concavity, it is found that the wavefront combining the convergence wavefront in the lens center and the divergence wavefront in both lens edges is observed. The $H$-plane far field radiation patterns with all lenses are shown in Fig. 14. The beamwidth of the main lobe in case of the modified lens without the concavity is almost the same as that in case of the topology optimized lens. However, it is found that the side lobe level with the modified lens enhances than that with the topology optimized lens.

Therefore, it is considered that the convergence effect by the hollow structure contributes the narrow beamwidth. Since the concave structure uniformizes the wavefront, it is expected to decrease the side lobe level. 


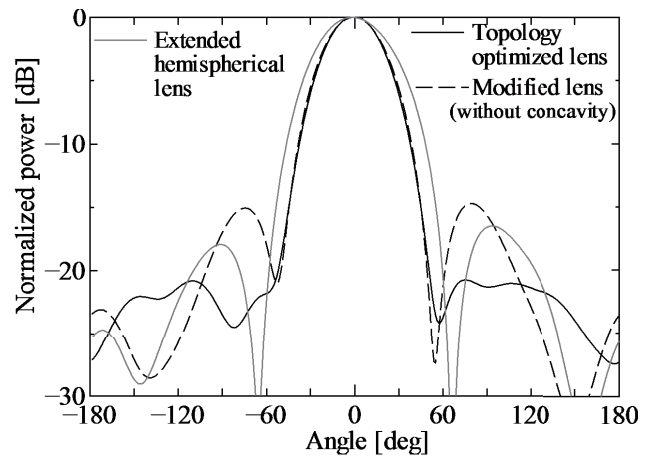

Fig. $14 H$-plane far field radiation patterns.

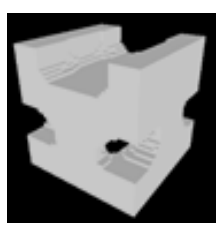

(a) OpenGL

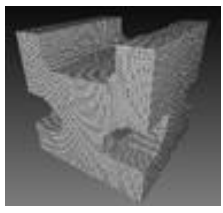

(b) STL format

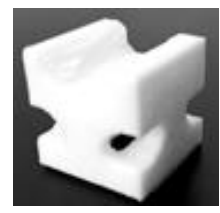

(c) Photograph
Fig. 15 CAD data and photograph of topology optimized lens.

\subsection{Manufacture and Measurement}

To confirm the effectiveness of the proposed method, the manufacture of the topology optimized lens and the far field radiation patterns measurement were carried out. Since the shape of the topology optimized lens becomes complex, the manufacture using the $3 \mathrm{D}$ printer is effective and low cost. FDM (fused deposition modeling) type 3D printer (MUTOH MF-500) and PLA (polylactic acid) as the filament material were used in this study. The design result to manufacture is recalculated by changing the relative permittivity, which is set to 2.6 assuming PLA. The objective function is same as the Eq. (4).

Due to manufacture the design results, it is necessary to convert the voxel data to the STL (standard triangulated language) format, which is one of data format of 3D CAD. Figure 15 (a) and (b) show the 3D display of the voxel data by the OpenGL and the 3D CAD viewer display by the STL format which is converted from the voxel data. The photograph of the topology optimized lens which was manufactured by the 3D printer is shown in Fig. 15 (c). Although the fabricated lens has some unevenness, it is reproduced faithfully based on the design data. The fabrication time was about 25 minutes. To prevent unnecessary voids into the lens, the deposition pitch is set as finely as possible.

The measurement results of the $H$-plane far field radiation patterns in case of the slot antenna loaded with the fabricated lens is shown in Fig. 16. For comparison, the radiation patterns without lens is also shown. Since the measurement results are in good agreement with the calculation results, it is found that the fabricated lens exhibits the performance as designed.

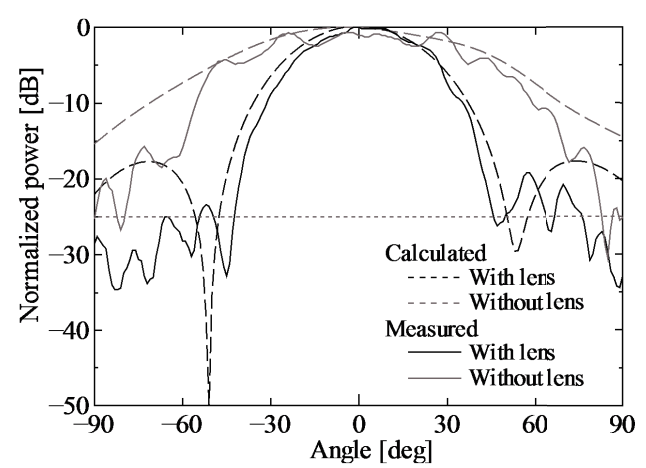

Fig. $16 \quad H$-plane far field radiation patterns.

By the manufacture and the measurement, it is shown that the proposed topology optimization method can design the dielectric lens shape, which is easy to manufacture. In addition, even with the complicated shape such as the hollow structure, it is possible to manufacture by the $3 \mathrm{D}$ printer.

\section{Conclusion}

We have applied the 3D topology optimization using the NGnet to the dielectric lens design for the slot antenna and have demonstrated that the proposed method has sufficient design performance. Especially, It is remarkable that the optimal lens has a hole in its inside. It would be difficult to find this kind of structure by designers and conventional optimization methods. The proposed method is applicable not only to the narrow angle lens but also to various applications such as a wide angle lens. The optimized results obtained by using a quarter basis model have shown symmetrical shapes, which can be manufactured by the 3D printer.

Future works will include the speed up of the proposed method and the application to the higher frequency device such as the millimeter wave antenna, the optical device, and so on.

\section{Acknowledgments}

This work was supported by JSPS KAKENHI Grant Number 15K06093, the Telecommunications Advancement Foundation, and the collaborative research program, information initiative center, Hokkaido University, Sapporo, Japan.

\section{References}

[1] G. Godi, R. Sauleau, and D. Thouroude, "Performance of reduced size substrate lens antennas for millimeter-wave communications," IEEE Trans. Antennas Propag., vol.53, no.4, pp.1278-1286, 2005.

[2] B. Schoenlinner, X. Wu, J.P. Ebling, G.V. Eleftheriades, and G.M. Rebeiz, "Wide-scan spherical-lens antennas for automotive radars," IEEE Trans. Microw. Theory Tech., vol.50, no.9, pp.2166-2175, 2002

[3] T. Shimizu and T. Yoneyama, "A NRD guide fed dielectric lens antenna with high gain and low sidelobe characteristics," IEICE Trans. Electron, vol.E88-C, no.7, pp.1385-1386, 2005. 
[4] K. Itoh, K. Miyata, and H. Igarashi, "Evolutional design of waveguide slot antenna with dielectric lenses," IEEE Trans. Magn., vol.48, no.2, pp.779-782, 2012.

[5] T. Nomura, K. Sato, K. Taguchi, T. Kashiwa, and S. Nishiwaki, "Structural topology optimization for the design of broadband dielectric resonator antennas using the finite difference time domain technique," Int. J. Numer. Meth. Eng., vol.71, no.11, pp.1261-1296, 2007.

[6] Y. Tsuji and K. Hirayama, "Design of optical circuit devices using topology optimization method with function-expansion- based refractive index destribution," IEEE Photon. Technol. Lett., vol.20, no.12, pp.982-984, 2008.

[7] K. Watanabe, F. Campelo, and H. Igarashi, "Topology optimization based on immune algorithm and multigrid methods," IEEE Trans. Magn., vol.43, no.4, pp.163-1640, 2007.

[8] T. Sato, K. Watanabe, and H. Igarashi, "Multimaterial topology optimization of electric machines based on normalized Gaussian network," IEEE Trans. Magn., vol.51, no.3, pp.1-4, 2015.

[9] J. Moody and C.J. Darken, "Fast learning in networks of locally-tuned processing units," Neural Computation, vol.1, no.2, pp.281-294, 1989.

[10] K. Watanabe, F. Campelo, Y. Iijima, K. Kawano, T. Matsuo, T. Mifune, and H. Igarashi, "Optimization of inductors using evolutionary algorithms and its experimental validation," IEEE Trans. Magn., vol.46, no.8, pp.3393-3396, 2010.

[11] R.J. Luebbers, K.S. Kunz, M. Schneider, and F. Hunsberger, "A finite-difference time-domain near zone to far zone transformation," IEEE Trans. Antennas Propag., vol.39, no.4, pp.429-433, 1991.

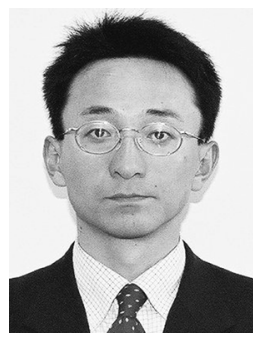

Keiichi Itoh received the B.S. and M.S. degrees in Electrical Engineering from Akita University in 1994 and 1996, respectively, and the $\mathrm{Ph} . \mathrm{D}$. degree in information science and electrical engineering from Hokkaido University in 2012. He is currently the associate professor in the National Institute of Technology, Akita College. His research interests include antenna and its application, electromagnetic analysis, and optimization design. He is a member of IEICE, JSST, International COMPUMAG Society, and

Japan AEM society.

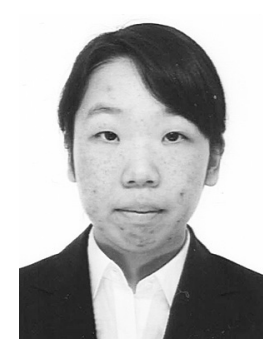

Haruka Nakajima is the student in the National Institute of Technology, Akita College. Her research interests include manufacture of dielectric lens by $3 \mathrm{D}$ printer.

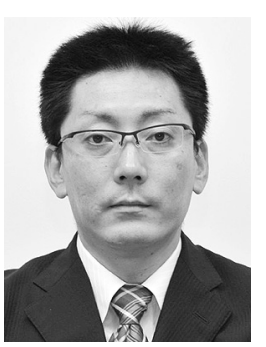

Hideaki Matsuda is currently the advanced technical officer in the National Institute of Technology, Akita College. His research interests include machine tool, manufacture of antenna and dielectric lens, millimeter-wave measurement.

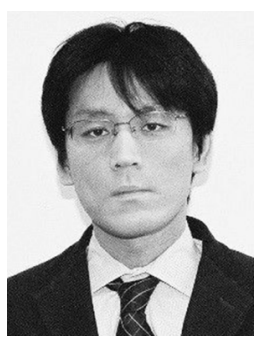

Masaki Tanaka received the B.S., M.S., and Ph.D. degrees in Electrical and Electronics Engineering from Akita University, Japan, in 1995, 1997 and 2001, respectively. He is currently the associate professor in the National Institute of Technology, Akita College, Japan. His research interests are millimeter wave passive devices and liquid crystal devices. He is a member of IEICE and the Japan Society of Applied Physics.

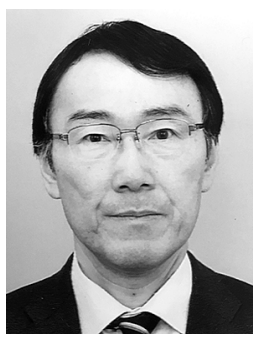

Hajime Igarashi received the B.E. and M.E. degrees in electrical engineering from Hokkaido University, Sapporo, Japan, in 1982 and 1984, respectively, and the $\mathrm{Ph} . \mathrm{D}$. degree in engineering from Hokkaido University in 1992. He has been a professor at the Graduate School of Information Science and Technology, Hokkaido University, since 2004. He was a guest researcher at Berlin Technical University, Germany, under support from the Humboldt Foundation from 1995 to 1997 . His research area is computational electromagnetism, design optimization and energy harvesting. He is a member of IEEJ, IEEE, Japan AEM society, JSST and International COMPUMAG society. He received culture, sports, science and technology minister's award and IEEJ distinguished paper award in 2016. 